elucidate the signalling pathway involving in heart remodelling of cTnI ${ }^{\mathrm{R} 146 \mathrm{~W}+/-}$ mice.

Methods Cardiac hypertrophy-related signalling pathway protein, such as calcineurin, calsarcin-1, GSK-3 $\beta$, AKT, SERCA2, PLB were detected by Western blot and RT-PCR. We also assessed the activity of calcineurin in $\mathrm{cTnI}^{\mathrm{R} 146 \mathrm{~W}+/-}$ mice, in order to elucidate potential mechanisms involving in the cardiac remodelling in $\mathrm{cTnI}^{\mathrm{R} 146 \mathrm{~W}+/-}$ mice.

Results The total expression of $\mathrm{cTnI}$ in $\mathrm{cTnI}^{\mathrm{R} 146 \mathrm{~W}+/-}$ mice was significant higher than $\mathrm{cTnI}^{\mathrm{R} 146 \mathrm{~W}-/-}$ mice $(\mathrm{p}<0.05)$, while the phosphorylation of $c T n I$ decreased significantly $(p<0.05)$, resulting in a obvious decrease of the ratio of phos-cTnI to $c \operatorname{TnI}(p<0.05)$. Pathological changes such as myocardial cell proliferation, cardiac hypertrophy, and interstitial fibrosis were observed by optical microscope in $\mathrm{cTnI}^{\mathrm{R} 146 \mathrm{~W}+/-}$ mice. Markers of cardiac hypertrophy, such as ANF, BNP, $\beta$-MHC increased significantly in $\mathrm{CTnI}^{\mathrm{R} 146 \mathrm{~W}+/-}$ mice $(p<0.05)$. The expression of calsarcin-1 in $\mathrm{cTnI}^{\mathrm{R} 146 \mathrm{~W}+/-}$ mice was significantly higher than that of $c \operatorname{TnI}^{\mathrm{R} 146 \mathrm{~W}-/-}$ mice $(\mathrm{p}<0.01)$, while other cardiac hypertrophy-related signalling pathway protein, such as calcineurin, GSK-3 $\beta$, AKT, SERCA2 did not change. The mRNA expression of PLB was reduced significantly by RT-PCR $(p<0.05)$. Meanwhile, the calcineurin activity of $\mathrm{cTnI}^{\mathrm{R} 146 \mathrm{~W}+/-}$ mice increased significantly $(p<0.01)$.

Conclusion $\mathrm{cTnI}^{\mathrm{R} 146 \mathrm{~W}+/-}$ mice had typical pathological cardiac remodelling and heart dysfunction, especially in the older ones. The expression of calsarcin-1 and the activity of calcineurin-NFAT signalling pathway may be the most important mechanism involving in pathological cardiac hypertrophy in $\mathrm{cTnI}^{\mathrm{R} 146 \mathrm{~W}-1-}$ mice.

\section{e0199 PERSIMIMON PEEL IMPROVED DYSLIPIDEMIA AND ITS RELATED PRODUCTION OF ATHEROGENIC AUTOANTIGEN COMPLEXES IN LOW-DENSITY LIPOPROTEIN RECEPTOR- DEFICIENT MICE}

doi:10.1136/hrt.2010.208967.199

OIAN Nanhu, Yang Zheng. First Affiliated Hospital of Jilin University, Changchun, China

Objective Roles of persimmon peel were investigated on possibility of developing atherosclerosis in low-density lipoprotein receptor (LDLR)-deficient mice in view of lipid metabolism, physico-biological oxidation, production of its related atherogenic autoantigen, and anti-atherogenic natural antibody production.

Method Male LDLR-deficient mice fed a high fat diet or a high fat diet supplemented with $10 \%$ dried and powdered persimmon peel (PP) for 12 weeks.

Result The PP supplementation significantly reduced the increment of plasma cholesterol and triglyceride levels. The high fat diet feeding increased plasma level of oxidised LDL/ $\beta 2$-glycoprotein I (oxLDL/ $/ 2 \mathrm{GPI}$ ) complexes as an atherogenic atuoantigen, while the PP supplementation significantly reduced the increment $(p<0.05)$. After the 12-week feeding, atherosclerotic plaque in the mice with the diet of PP decreased by $70 \%$ as compared to that in mice fed the high fat diet $(\mathrm{p}<0.005)$. The PP feeding also reduced urinary 11dehydrothromboxane $\mathrm{B} 2$, a stable metabolite of platelet activation marker thromboxane A2, but IgM level of anti-oxLDL antibodies was not changed.

Conclusion Thus, these results demonstrate that persimmon peel may have an anti-atherogenic property through normalisation of lipid metabolism and may be able to reduce production of the atherogenic complexes

\section{e0200 EFFECTS OF MESENCHYMAL STEM CELLS ON MATRIX METALLOPROTEINASE SYNTHESIS OF CARDIAC FIBROBLASTS}

doi:10.1136/hrt.2010.208967.200

Ya-Ping Wang, Xin-Yang Hu, Xiao-Jie Xie, Jian-An Wang. Second Affiliated Hospital Zhejiang University College of Medicine, Hangzhou, China

Objectives Mesenchymal stem cell (MSC) transplantation has been known to decrease matrix metalloproteinase (MMP) synthesis in the myocardium after myocardial infarction (MI) and to improve ventricular remodelling. However, the underlying mechanisms behind MSC have not been clearly demonstrated yet. This study investigated the effects of MSCs through paracrine actions on the MMP synthesis of cardiac fibroblasts (CFs).

Methods CFs were placed under hypoxia conditions for $24 \mathrm{~h}$ before co-culture with MSCs or hypoxia preconditioning MSCs (HPMSCs) in transwell. CFs and MSCs/HP-MSCs shared the same medium, in which erythropoietin (EPO) antibody and EPO receptor (EPOR) were/were not added. Gelatin Zymography was used to detect the gelatinolytic activity of matrix metalloproteinase-2 (MMP-2) and matrix metalloproteinase-9 (MMP-9) in culture media of CFs with different conditions. Western-Blotting was used to assay MMP-2, MMP-9 and TIMP-1 synthesis of CFs. The ERK1/2 signalling pathway was also investigated.

Results Protein expression and activity of MMP-2 produced by CFs significantly increased by about 1.4 -fold $(p<0.01)$ through hypoxia and decreased after co-culture with MSCs or H-MSCs. This is not the case with MMP-9. Mediation of effects may involve phosphorylation of ERK1/2. Tissue inhibitors of metalloproteinases-1 (TIMP-1) had reverse effects on regulation of MMP-2. Either exogenous EPOAb or EPOsR partially inhibited MSCs effect on MMP-2 protein expression and activity by CFs.

Conclusions MSCs may influence MMP/TIMP expression by CFs via the ERK1/2 pathway and EPO may acts as a key factor in the paracrine actions of MSCs.

\section{C0201 HEAT SHOCK PROTEIN 90 PROTECTS RAT MESENCHYMAL STEM CELLS AGAINST HYPOXIA AND SERUM DEPRIVATIONINDUCED APOPTOSIS VIA PI3KAKT AND ERK12 PATHWAYS}

doi:10.1136/hrt.2010.208967.201

Gao Feng, Hu Xinyang, Xie Xiaojie, Xu Oiyuan, Wang Yaping, Liu Xianbao, Xiang Meixiang, Sun Yong, Wang Jian-an. Second Affiliated Hospital, Zhejiang University College of Medicine, Hangzhou, China

Objective Mesenchymal stem cells (MSCs) transplantation has shown therapeutic potential to repair the ischaemic and infracted myocardium, but the effects are limited by apoptosis and loss of donor cells in host cardiac microenvironment. The aim of this study is to explore the cytopretection of Hsp90 against hypoxia and serum deprivation induced apoptosis and the possible mechanisms.

Methods Cell viability was determined by 3-(4.5-dimethylthiazol-2yl)-2, 5-diphenyltetrazolium bromide assay. Apoptosis was assessed by Hoechest 33258 nuclear staining and flow cytometric analysis with annexin V/PI staining. The gene expression of TLR4 and ErbB2 was detected by real-time PCR. The protein levels of cleavedcaspase3, bcl-2, bcl-xL, bax, total-Erk, phospho-Erk, total-Akt, phospho-Akt and hsp90 were detected by western-blot. The production of nitric oxide was measured by spectrophotometric assay.

Results Hsp90 improves MSCs viability and protects MSCs against apoptosis induced by serum deprivation and hypoxia. The 
protective role of Hsp90 not only elevates bcl-2/bax and bcl-xL/bax expression but also decrease cleaved-caspase3 expression via downregulating TLR-4 and ErbB2 membrane receptors. By binding to TLR-4 and ErbB2, Hsp90 activates the PI3K/Akt and ERK1/2 pathways. Hsp90 also down regulates the pro-apoptotic protein bax. It is demonstrated that exogenous Hsp90 elevates the expression levels of bcl-2/bax and bcl-xL/bax by activating the TLR-4 and ErB2 downstream PI3K/Akt and ERK1/2 pathways, which decreases cleaved caspase-3.

Conclusion Hsp90 significantly protects MSCs against apoptosis induced by hypoxia and serum deprivation. These findings demonstrates a novel and effective treatment strategy against MSC apoptosis in cell transplantation.

\section{e0202 EFFECTS OF RANOLAZINE ON ACTION POTENTIAL AND CONTRACTION FORCE IN GUINEA PIG PAPILLARY MUSCLES}

doi:10.1136/hrt.2010.208967.202

Guotao Wang, Ping Yang. Department of Cardiology, China-Japan Union Hospital of Jilin University, Changchun, China

Objective To observe the effects of ranolazine on the action potential and contraction force in guinea pig papillary muscles. To explore the mechanism of ranolazine anti-arrhythmia and myocardial ischaemia.

Methods 18 healthy adult guinea-pigs were randomly divided into $\mathrm{H}_{2} \mathrm{O}_{2}(200 \mathrm{mmol} / \mathrm{l})$ groups, ranolazine $(10 \mathrm{mmol} / \mathrm{l})+\mathrm{H}_{2} \mathrm{O}_{2}$ groups and TTX $(2 \mathrm{mmol} / \mathrm{l})+\mathrm{H}_{2} \mathrm{O}_{2}$ groups, with six guinea pigs in each group compared before and after administration to observe the effects of ranolazine on the papillary muscles.

Results $\mathrm{H}_{2} \mathrm{O}_{2}$ could increase action potential durations measured at $50 \%$ repolar... moreization levels and $90 \%$ repolarisation levels were prolonged $(p<0.001)$. There was reduced myocardial contractility $(p<0.05)$ in contraction force in the guinea pigs compared to before administration. Ranolazine can inhibit action potential durations measured at the $50 \%$ repolar... moreization levels and the $90 \%$ repolarisation levels were prolonged by $\mathrm{H}_{2} \mathrm{O}_{2}$, but the effect was weaker compared to that of TTX. Ranolazine and TTX could improve myocardial contractile force by reducing the $\mathrm{H}_{2} \mathrm{O}_{2}$-induced. Conclusion ranolazine could reduce action potential duration the $\mathrm{H}_{2} \mathrm{O}_{2}$-induced and increase contraction force. TTX performs a similar role.

\section{e0203 ATHEROSCLEROSIS IMPAIRS CXCR4 EXPRESSION AND FUNCTION IN BONE MARROW CELLS}

doi:10.1136/hrt.2010.208967.203

Xu Qiyuan, Yu Hong. Second Affiliated Hospital, Zhejiang University College of Medicine, Hangzhou, China

Objectives The chemokine stromal cell-derived factor-1 (SDF-1) plays a critical role in mobilising CXCR4-positive precursor cells in the bone marrow and may be essential for efficient vascular regeneration and repair. We recently reported that CXCR4 and the angiogenic potential of bone marrow derived cells (BMCs) were regulated positively by calcium and negatively by ageing. We hypothesise that atherosclerosis may also affect CXCR4 surface expression and functions. This study is to determine that atherosclerosis defects BMCs both on the decreased population of CXCR4 + progenitor cells and on the impaired mobility as well as to explore possible mechanisms.

Methods We established atherosclerosis model on ApoE-/- mice under at least 6-month High Fat Diet (group Ath), and used 3 4week aged ApoE-/- mice as control (group Ctrl). In order to evaluate impact of the disease on CXCR4 expression from gene transcription to protein synthesis, surface CXCR 4 expression on BMCs from the two groups of mice were analysed with FCM; the mRNA of CXCR4 was detected by real-time PCR. To compare the inducibility of CXCR4 expression in response to environmental change, $1 \mathrm{mM}$ $\mathrm{CaCl}_{2}$ was applied as an extracellular stimuli to treat BMCs for $4 \mathrm{~h}$. To examine how $\mathrm{BMC}_{\text {Ath }}$ are defect on calcium-induced CXCR4 surface expression, calcium influx was analysed by measuring the increase of intracellular calcium after BMCs were mixed with $\mathrm{CaCl}_{2}$. Cell-cell adhesion assays were used to detect the SDF-1 mediated adhesion of BMCs to the HUVEC monolayer and vertical invasion assays were used respectively to quantify trans-endothelial migration of BMCs in response to a gradient of SDF-1.

Results $\mathrm{BMC}_{\mathrm{Ath}}$ have less number of cells expressing CXCR4 as compared to $\mathrm{BMC}_{\mathrm{Ctrl}}(8.54 \pm 1.96 \%$ vs $13.75 \pm 3.94 \% ; \mathrm{n}=10, \mathrm{p}<0.01)$ CXCR4 expression on $\mathrm{BMC}_{\mathrm{Ctrl}}$ could be enhanced by calcium, but CXCR4 surface expression in $\mathrm{BMC}_{\mathrm{Ath}}$ increased significantly lesser then $\mathrm{BMC}_{\mathrm{Ctrl}}(11.24 \pm 1.31 \%$ vs $26.59 \pm 4.92 \% ; \mathrm{n}=10, \mathrm{p}<0.01)$. It is partly because of the defective calcium influx in $\mathrm{BMC}_{\text {Ath }}$ which reduced the CXCR4 gene transcription, consequently leading to impaired responses on calcium-induced CXCR4 surface expression. $\mathrm{BMC}_{\text {Ath }}$ showed weaker lower mobility and lower trans-endothelial migration ( $0.80 \pm 0.11 \mathrm{~mm}$ vs $1.17 \pm 0.15 \mathrm{~mm} ; \mathrm{n}=4, \mathrm{p}<0.05)$, and this was not enhanced by calcium pretreatment.

Conclusions Atherosclerosis impairs CXCR4 surface expression on BMCs and related cell functions.

\section{e0204 HEAT SHOCK PROTEIN 90 ENHANCES RAT MESENCHYMAL STEM CELLS MIGRATION VIA PI3KAKT AND ERK12 PATHWAYS}

doi:10.1136/hrt.2010.208967.204

Gao Feng, Hu Xinyang, Xie Xiaojie, Xu Qiyuan, Wang Yaping, Liu Xianbao, Wu Rongrong, Xiang Meixiang, Sun Yong, Wang Jian-an. Second Affiliated Hospital, Zhejiang University College of Medicine, Hangzhou, China

Objective Heat shock protein 90 (HSP90) is a chaperone for several client proteins involved in transcriptional regulation, signal transduction, and cell cycle control. HSP90 is abundantly expressed by a variety of tumour types and has been recently targeted for cancer therapy. The objective of this study is to determine the role of Hsp90 in regulating the migration of Mesenchymal stem cells and to determine the mechanism. We hypothesised that inhibition of Hsp90 impairs the MSCs migration via PI3K/Akt and ERK1/2 signalling pathways.

Methods The MSCs were cultured from femoral and tibia. The ability for MSCs cells to migrate is to be determined by the wound healing assay and transwell assay. The activity of matrix metalloproteinase-2 (MMP-2) and matrix metalloproteinase-9 (MMP-9) were estimated by gelatin -zymography. The mRNA levels of MMP-2, MMP-9, CXCR4 and VCAM-1 were detected by real-time PCR. The protein expression of MMP-2, MMP-9 and ERK1/2, phospho-ERK1/2, Akt and phospho-Akt were determined by Western-blot

Results Treatment with RhHsp90 $\alpha$ significantly enhances MSCs migration from $9.83 \pm 2.48$ to $48.65 \pm 2.81$ cells. Treatment with sirhsp $90 \alpha$ significantly decreased MSCs migration compared with treatment of hsp90 $\alpha$ from $63.33 \pm 9.61$ to $13.00 \pm 4.38$ cells. Pretreat with 17-AAG, wortmannin, U0126, decreased MSCs migration to $13.33 \pm 1.29,15.33 \pm 2.1,16.5 \pm 3.3$ cells, respectively. Treatment with RhHsp90 $\alpha$ enhanced the MSCs secretion of MMP-2 and MMP-9, as well as significantly increasing the activity of MMP-9, and increasing the expression of CXCR4 and VCAM-1. PI3K/Akt and ERK signalling pathways mediate the promotion of MSCs migration by RhHsp90a. 